\title{
El síndrome de desgaste profesional (burnout syndrome) Burnout syndrome
}

\author{
Arturo Mendoza López* \\ * Psicoanalista por el Instituto Mexicano de Psicoanálisis, con sede en: Asociación Psicoanalítica Mexicana. Hospital ABC. \\ Citar como: Mendoza LA. El síndrome de desgaste profesional (burnout syndrome). \\ Neurol Neurocir Psiquiatr. 2021; 49 (2): 73-83. https://dx.doi.org/10.35366/103355
}

\section{INTRODUCCIÓN}

Lo más sorprendente que encontré al hacer la investigación acerca de esta condición, síndrome o este trastorno es que no existe; o sea, que hay un "chance" de que alguno de ustedes decida irse, ya que quizá no querrán oír de algo que no existe. No existe por lo menos oficialmente en el DSM-5, lo cual me pareció increíble, por la tremenda importancia que espero ustedes puedan ver en los siguientes minutos.

Como ustedes oirán más adelante, la Dra. Anita Everett hizo de su presidencia en la Asociación Psiquiátrica Americana un escaparate para este problema. Entonces le hablé, y me refirió al departamento de investigaciones de la APA. El departamento de investigación de la Asociación Psiquiátrica Americana (Stephanie Smith) me hizo el favor de mandarme la siguiente aclaración: aunque específicamente no existe como tal, sí existe, escondido, sepultado en esos códigos oscuros bajo la rúbrica "Z", la cual incluyen los "problemas ocupacionales" (específicamente el Z56.9 "otros problemas relacionados al empleo") (Figura 1). Esto está localizado bajo el rubro de "otras condiciones que pueden ser el foco de atención clínica". Este código incluye "el empleo o el medio ambiente de trabajo"; incluye "la falta de trabajo, los cambios recientes de trabajo, amenazas de pérdida del trabajo, insatisfacción laboral, horarios estresantes en el trabajo, incertidumbre acerca de las opciones vocacionales, ser molestado sexualmente en el trabajo, problemas personales con el jefe, supervisor, colaboradores u otras personas en el ambiente laboral; medio ambiente de trabajo hostil u otros estresores psicosociales relacionados con el trabajo; o cualquier otro problema relacionado con el trabajo o la ocupación". ${ }^{1}$

También bajo el código Z73.0 en la Clasificación Internacional de Enfermedades, ${ }^{2}$ está uno de los síndromes menos conocido para ser considerado como real o válido y para ser tomado en cuenta. Pero esto no quiere decir que no sea un tema de suma importancia, sobre todo para nosotros los profesionistas.

Hay que aclarar que en varios países europeos no sólo se diagnostica como tal, sino que los seguros médicos pagan su tratamiento. Lo que lo ha hecho visible es "la lana", ya que se ha estimado, conservadoramente, que en EU se pierden por lo menos 5 billones de dolaras anuales, y desde luego que es muy común. ${ }^{3}$

\section{DESVENTAJAS}

Quizá una desventaja es que anteriormente se le consideraba como "neurastenia", término pasado de moda (ICD-10, código F48.0). ${ }^{2}$ Otra desventaja es que se puede considerar como una debilidad de carácter. También se le ha visto como algo que fácilmente se debería de haber prevenido. Como su nombre lo indica, se describe en profesionistas que deberían tener la fortaleza para no caer tan bajo. Este diagnóstico tiene un gran estigma.

Como muchas veces estos profesionistas forman parte de un equipo, a los otros no les conviene no tener las funciones de los desvalidos y muestran poca empatía. Esta falta de empatía y de apoyo al caído por parte del equipo que debería de asistir es muy común, no consciente y muy desmoralizadora y desilusionante para el que trae "las pilas bajas". Esto aumenta la sensación de aislamiento y de disociación. 
Otra cosa que ocurre, sobre todo en EU, es que cuando baja la competitividad del "quemado", éste puede empezar a realizar actividades con errores, los cuales pueden ser reportados a los consejos médicos y, en ocasiones, se pierden los trabajos, se les restringe la licencia o hasta se les revoca, aumentando la desesperación del afectado. Los consejos médicos para reinstalar la licencia tienen que preguntar al que solicita si ha padecido un problema psiquiátrico, si ha hecho uso de sustancia o ha estado bajo tratamiento psiquiátrico, y esto complica aún más la situación del afectado. En EU 400 médicos se suicidan anualmente (Figura 2).

\section{LO BÁSICO Y LA NOMENCLATURA}

Principalmente, el problema es haber padecido un estrés (distrés) por un tiempo demasiado prolongado. Este síndrome también es conocido como "burnout" pero también tiene otros nombres: "síndrome de trabajador desgastado", "síndrome del trabajador consumido", "síndrome del trabajador quemado" o "síndrome de desgaste ocupacional"; con todo y que se le conoce con tantos nombres, se le hace poco caso. Se tiene que diferenciar del "trauma vicariante" y de la "fatiga por compasión" (que los psiquiatras y otros médicos sufrimos por ejercer empatía), aunque pueden estar juntos.

\section{CONFESIONES PERSONALES (H. STEVEN MOFFIC)}

Generalmente se dice que todo escrito es autobiográfico. Acepto o confieso desde un inicio que este escrito es descaradamente autobiográfico: yo lo he padecido y me propongo a mí mismo como un ejemplo de este padecimiento. Creo que lo empecé a padecer hace cuatro o cinco años y afortunadamente me estoy recuperando. Ocurrió cuando era Presidente de la Sociedad Mexicana de Neurología y Psiquiatría. Anteriormente, hace años, yo había trabajado mucho más — pero mucho más - pues, al mismo tiempo, a finales de los ochenta fui Presidente de la Asociación Mexicana de Psiquiatría Infantil y Jefe del Departamento de Psiquiatría y Medicina del Adolescente del Hospital Infantil de México "Federico Gómez"; organicé varios congresos nacionales $y$, al mismo tiempo, era Presidente de mi colonia, además de otros cargos; tenía una consulta privada llenísima, era el administrador del Grupo

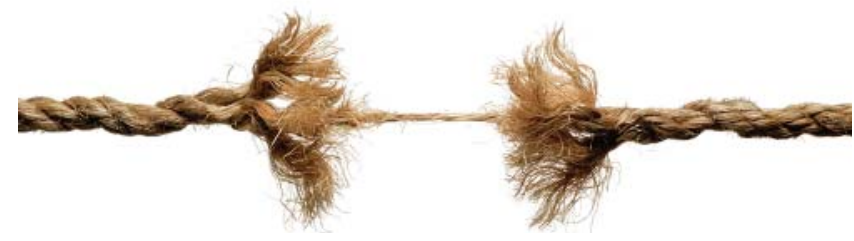

Figura 1.

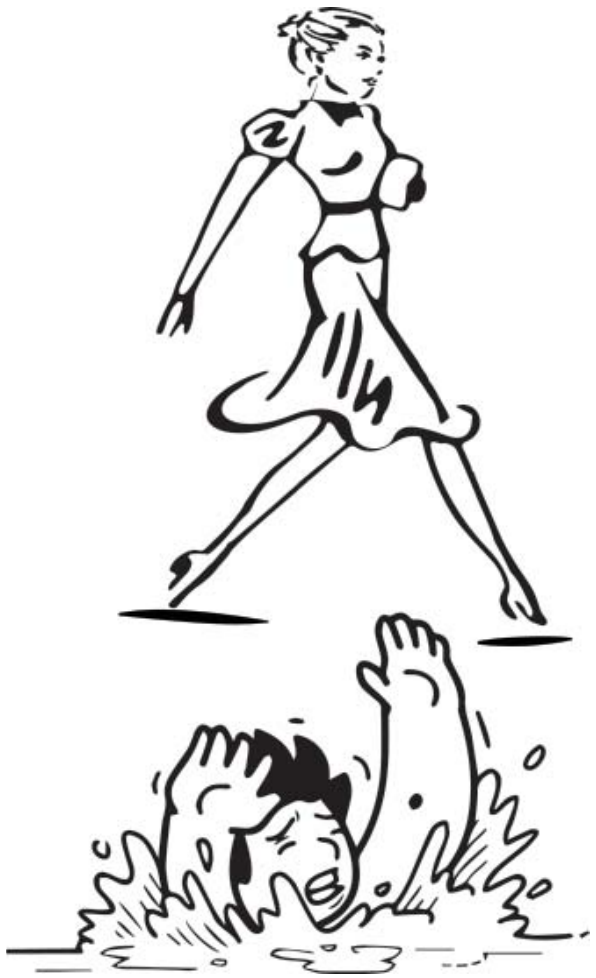

Figura 2.

Psiquiátrico Infantil de México; en esa época mi padre murió, compré una casa que remodelé y a la que monitorizaba diariamente $y$, además me casé. Con todo y todo no tuve un desgaste anormal. Pero eso fue hace 30 años; la edad es un factor que lo facilita. Definitivamente: "no es lo mismo los tres mosqueteros que 30 años después".

En esa época empecé a aprender a decir que no. Cuando uno tiene muchos cargos se le acumulan las invitaciones a, por ejemplo, dar una plática u organizar un congreso, etc. Llegó el momento en que me estaba medio volviendo loco porque a todo decía que sí. Aunque les parezca ridículo practicaba enfrente del espejo a decir que no: "No gracias", "Les agradezco mucho, pero en este momento no puedo hacerlo", "Que amables son por pensar en mí, pero tengo que declinar su invitación", etc., técnicas cognitivas-conductuales que yo conocía desde hace tres o cuatro décadas.

\section{ETAPAS DE ESTRÉS, DIBUJO DE SÍNTOMAS (Figuras 3 y 4 )}

\section{Sintomatología}

Parte de lo que nos pasa a los que hemos sufrido este desgaste es que literalmente perdemos la sensación del tiempo, dejamos de concientizar - aunque parezca locoque el día sólo tiene 24 horas y que parte de éstas deben ser usadas en dormir, comer, socializar, gozar de la fami- 


\section{CONTINUIDAD DEL ESTRÉS}
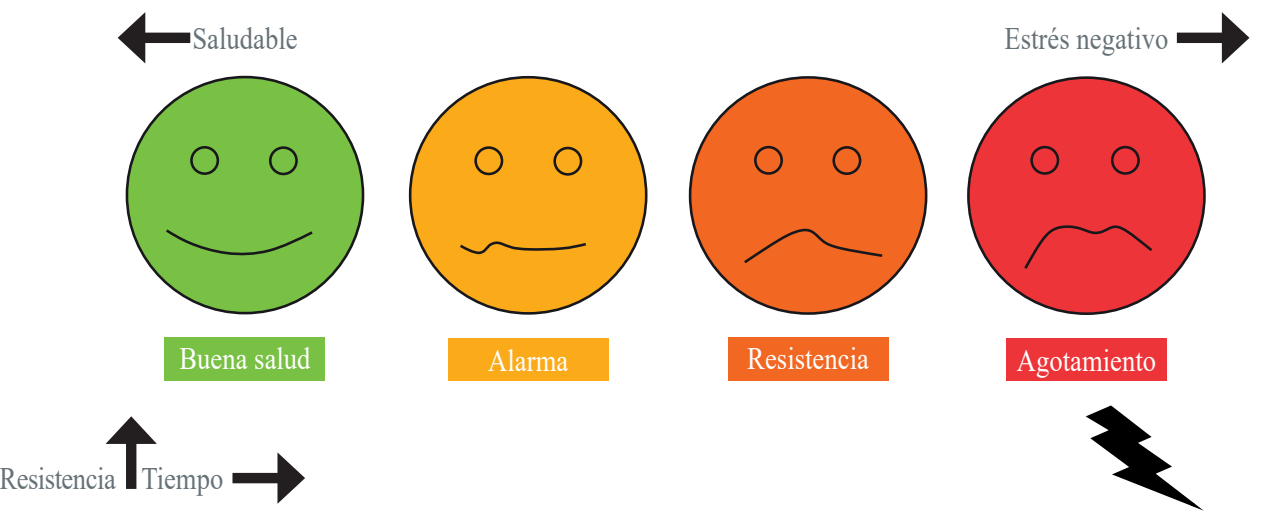

Figura 3.

lia, etc., y no sólo para trabajar, aunque no lo hacemos. Desde luego tenemos que aceptar que muchos tenemos una adicción al trabajo.

Los síntomas del desgaste profesional son muy simples: cansancio, desinterés en actividades que anteriormente se realizaban aún con entusiasmo, despersonalización, sensación de desorganización mental, de aislamiento afectivo e interaccional, sensación de que "uno no puede con el paquete"; insatisfacción y angustia, trastornos del sueño; síntomas depresivos, ideación suicida y aun actos suicidas. Existe una progresiva pérdida del idealismo y energía. Se sigue con un cinismo. Definitivamente se pierde el bienestar en todas las áreas y la misma conexión consigo mismo y con los demás que eran antes cercanos. Se empieza a perder el significado de la vida misma. Podemos estar irritables.

Lo principal es un fuerte sentimiento de impotencia, ya que, desde el momento de levantarse, ya se siente uno mismo cansado. El trabajo no tiene fin $y$, a pesar de que se hace todo para cumplir con los compromisos, el trabajo nunca se termina. La persona que lo padece se vuelve anhedónica, es decir, que lo que anteriormente era motivo de alegría ahora no lo es, en otras palabras, pierde la capacidad de disfrutar. Aun cuando se tiene tiempo, se siente siempre estresado. A diferencia de lo que ocurría al principio, el trabajo ya no produce incentivos para la persona afectada con desgaste profesional. Visto por otras personas, aparenta hipersensibilidad, depresión e insatisfacción, múltiples y variados síntomas físicos, espirituales, económicos y familiares y efluvio telogénico (caída del cabello en los lavados o al peinarlo).

\section{A PUNTO DE SUICIDARSE (Figura 5)}

\section{¿Cómo y cuándo empieza?}

Desde el punto de vista del desarrollo, el problema puede empezar desde chico y se puede ver cómo se va desarro-

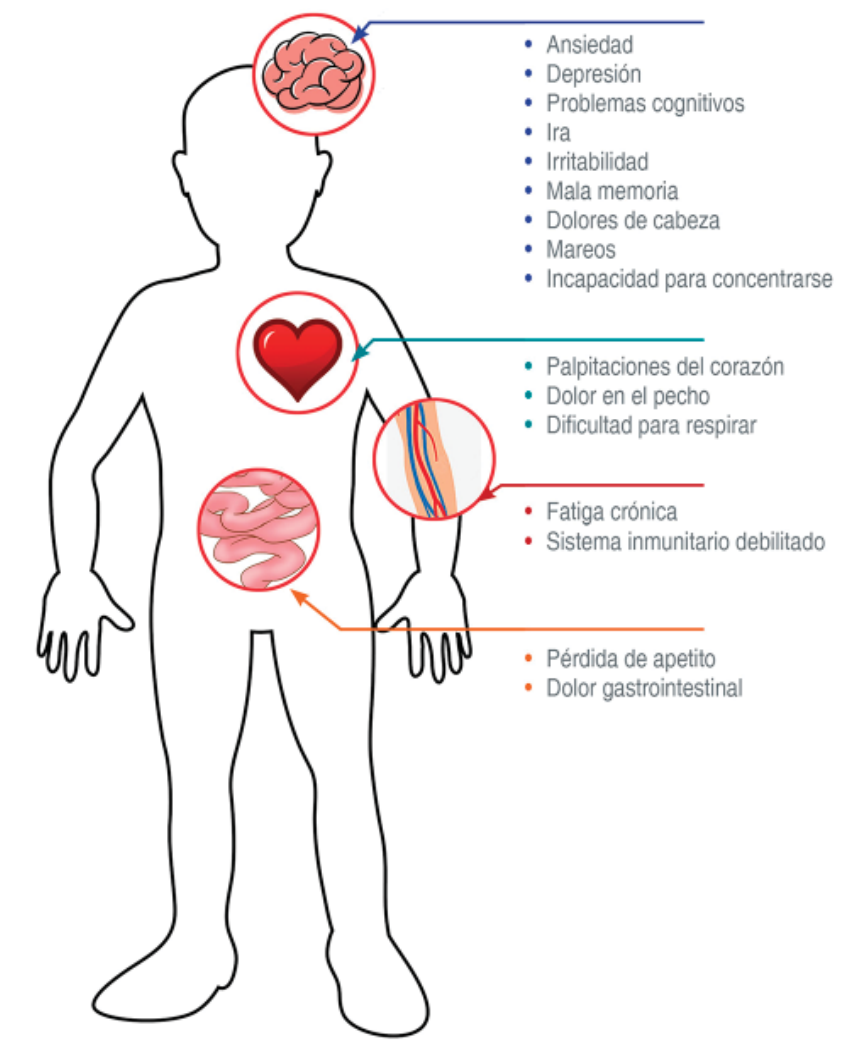

Figura 4.

Ilando lo que uno espera de sí mismo. Aquí es donde el psicoanálisis ayuda entender los orígenes, si vemos el tema del ideal del yo, y "chance" un poquito el superyó.

Este desgaste profesional puede empezar en los médicos desde que empiezan a estudiar la carrera de medicina; definitivamente durante el internado, el 76\% de los residentes lo padecen. Yo aprendí a dormir caminando durante el internado. 


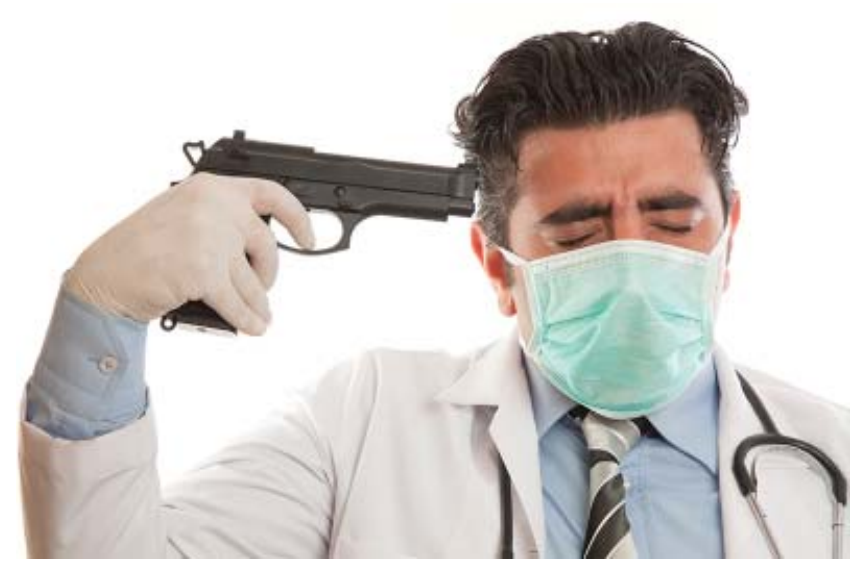

Figura 5.

La Clínica Mayo y la Asociación Médica Americana realizaron un estudio de 6,880 médicos y encontraron que aproximadamente $45 \%$ lo padecían. En un seguimiento que se hizo después del 2014 se encontró que 54\% tenían por lo menos un signo de desgaste profesional. Se usó el Maslach Burnout Inventory. Además de la insatisfacción profesional, se producen muchos síntomas físicos, psicológicos, interaccionales, espirituales y económicos que veremos a continuación. Inicialmente hay un estado de negación por el sistema de profesionalismo, perfeccionismo, altruismo, idealismo, adicción al trabajo y de hiperresponsabilidad.

De nuevo, básicamente, es una reacción al distrés crónico, que no se ha podido elaborar. Algo que el sujeto cree que puede superar o que no le queda otra por sus propias ambiciones, sensación de compromiso, apego emocional e idealismo.

\section{BREVE HISTORIA E IMPORTANCIA}

Increíblemente este padecimiento fue apenas descrito en 1974 por Freudenberger, aunque ya había sido descrito cinco años antes, pero hablando del desgaste policial. El término de "burnout" ${ }^{\text {" }}$ se originó en la literatura hace un siglo, al describir el fenómeno que les ocurría a los trabajadores sociales de Nueva York que eran muy dedicados, inteligentes y comprometidos con su trabajo, pero con demasiadas expectativas imposibles de cumplir. No se daban por vencidos, aunque no tenían un grupo de apoyo ni supervisión.

Hay múltiples explicaciones desde la teoría psicoanalítica a la social que no voy a incluir aquí ya que ocuparía mucho tiempo (Wikipedia). Tampoco me meteré en las discusiones de varios autores entre estrés simple y el desgaste profesional. Los japoneses tienen el término de karoshi para explicitar la muerte por exceso de trabajo.

Afortunadamente la expresidenta de la Asociación Psiquiátrica Americana, la Dra. Anita Everett, se interesó en el tema y promovió que se escriban artículos en el Psychiatric News. En dicha asociación existe todo un capítulo que se encarga del "wellbeing" y el "burnout" para los psiquiatras. Obviamente allá sí se han dado cuenta del gran problema que es y han trabajado estructuradamente para proteger a los psiquiatras.

Por ejemplo, en un artículo llamado "Superando el Burnout", ${ }^{4}$ la Dra. Constance Guille, miembro de dicho capítulo, escribe acerca de los abordajes que se han tratado para aliviar este síndrome. Ella cita cómo la Asociación Médica Americana junto con los principales diez sistemas de cuidados de salud declararon en marzo del 2017 que el burnout en médicos era una crisis de salud pública.

\section{LOGO DEL CAPÍTULO DEL WELL-BEING Y BURNOUT DE LA APA (Figura 6)}

¿Qué médicos se desgastan más? (Tabla 1).

¿Qué médicos se desgastan y se deprimen? (Tabla 2).

El $42 \%$ se desgasta tanto si trabajas independientemente o asalariado.

¿Qué contribuye a la depresión de los doctores? (Tabla 3).

La depresión sí afecta a los pacientes (Tabla 4).

La depresión afecta la relación con nuestros colegas (Tabla 5).

¿Qué contribuye al desgaste? (Tabla 6).

\section{Etapas}

1) Compulsión de probarse a sí mismo, ambición excesiva.

2) Tratar de trabajar más duro.

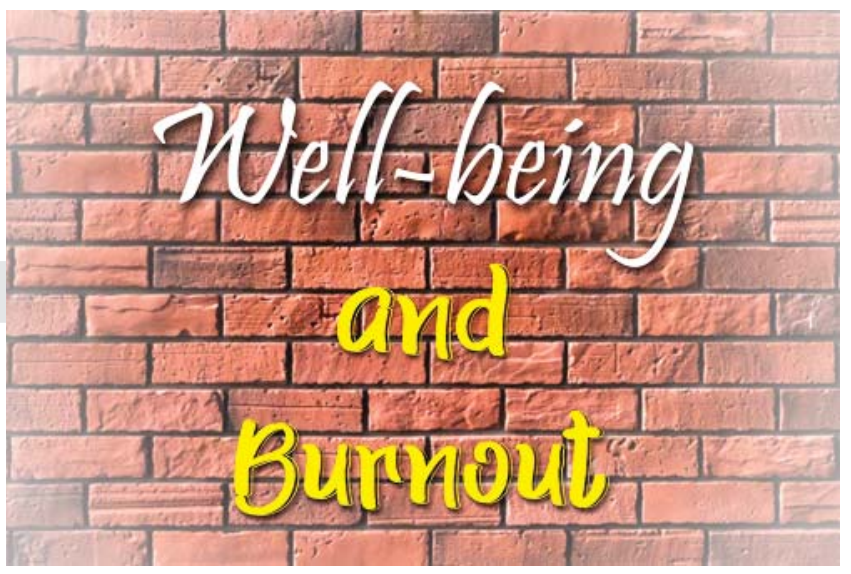

Figura 6. 
Tabla 1: ¿Qué médicos son los más agotados?

\begin{tabular}{ll}
\hline Especialidad médica & $\%$ \\
\hline Medicina Crítica & 48 \\
Neurología & 48 \\
Medicina Familiar & 47 \\
Obstetricia/Ginecología & 46 \\
Medicina Interna & 46 \\
Medicina de Emergencia & 45 \\
Radiología & 45 \\
Medicina Física y Rehabilitación & 44 \\
Urología & 44 \\
Alergias e Inmunología & 44 \\
Cirugía General & 43 \\
Cardiología & 43 \\
Otorrinolaringología & 42 \\
Medicina Pulmonar & 41 \\
Pediatría & 41 \\
Enfermedades Infecciosas & 40 \\
Nefrología & 40 \\
Oncología & 39 \\
Gastroenterología & 38 \\
Anestesiología & 38 \\
Reumatología & 38 \\
Psiquiatría & 36 \\
Salud Pública y Medicina Preventiva & 36 \\
Diabetes y Endocrinología & 35 \\
Ortopedia & 34 \\
Oftalmología & 33 \\
Patología & 32 \\
Dermatología & 32 \\
Cirugía Plástica & 23 \\
\hline
\end{tabular}

Fuente: Medscape.

3) Empezar a ser negligente con las necesidades personales (dormir, comer, descansar, recreaciones, familia).

4) Sobrevaluar el trabajo sobre todo lo demás.

5) Negación del problema, pensar que los demás son flojos.

6) Dejar de socializar.

7) Cambios conductuales que son observados por otros (irritabilidad).

8) Separación de sí mismo y de otros.

9) Sensación de vacío interno.

10) Depresión.

11) Síndrome de desgaste emocional bien establecido con colapso físico y mental.

\section{CAUSAS Y CONSECUENCIAS}

Terminemos primero por las causas y consecuencias de este síndrome. Hace un momento les dije uno de los factores que facilitan este padecimiento: edad avanzada. ${ }^{5}$ En la Asociación Psiquiátrica Americana existe una organización que junta y ayuda a los seniors psiquiatras. El Dr. Wick advierte que puede llegar el momento en el que la edad avanzada y una disminución de las capacidades profesionales se pueden juntar. Él aconseja ir preparando el retiro en una manera juiciosa.

Desde luego el trabajar mucho sin descansar lo suficiente es la principal causa. Otro elemento que ya mencioné es la falta de apoyo y empatía. Siento que como entes sociales que esperan trabajar en equipo repartiendo equitativamente responsabilidades, pero que no ven cumplidas estas expectativas, se percibe una sensación de aislamiento y aun de traición. Estos últimos aspectos son descuidados pero importantes.

Las características de personalidad desde luego son esenciales: caracteres perfeccionistas, idealistas, obsesivos, heroicos y sobre todo estoicos. El sistema-medioambiente alrededor es muy demandante, exigente y a veces frío, distante, corporativista y mercantilista.

El primer paso para la recuperación es algo que se dice fácil, pero que no lo es, y tampoco el darse cuenta de la situación. No es fácil porque generalmente es progresivo y lento. A

Tabla 2: ¿Qué médicos padecen depresión y agotamiento?

\begin{tabular}{lc}
\hline Especialidad médica & $\%$ \\
\hline Obstetricia/Ginecología & 20 \\
Salud Pública y Medicina Preventiva & 18 \\
Urología & 17 \\
Neurología & 17 \\
Medicina Familiar & 16 \\
Medicina Crítica & 16 \\
Radiología & 16 \\
Medicina Interna & 15 \\
Medicina Física y Rehabilitación & 15 \\
Cirugía General & 15 \\
Diabetes y Endocrinología & 15 \\
Patología & 14 \\
Ortopedia & 14 \\
Cardiología & 13 \\
Alergias e Inmunología & 13 \\
Otorrinolaringología & 13 \\
Anestesiología & 13 \\
Oncología & 13 \\
Medicina Pulmonar & 12 \\
Medicina de Emergencia & 12 \\
Pediatría & 11 \\
Gastroenterología & 11 \\
Reumatología & 10 \\
Cirugía Plástica & 10 \\
Enfermedades Infecciosas & 9 \\
Dermatología & 9 \\
Oftalmología & 9 \\
Nefrología & 9 \\
Psiquiatría & 8 \\
\hline
\end{tabular}

Fuente: Reporte Medscape Nacional sobre Depresión y Agotamiento en Doctores 2018. 
Tabla 3: ¿Qué contribuye a la depresión de los doctores?

\begin{tabular}{lcc}
\hline Situación & \% Mujeres & \% Hombres \\
\hline Trabajo & 5.6 & 5.6 \\
Finanzas & 3.7 & 3.9 \\
Familia & 3.2 & 3.1 \\
Relaciones románticas & 3.1 & 2.9 \\
Salud & 2.9 & 2.9 \\
\hline
\end{tabular}

Tabla 4: ¿Su depresión afecta la atención al paciente?

\begin{tabular}{lc}
\hline & $\%$ \\
\hline Mi depresión no afecta mi & 40 \\
interacción con los pacientes & 33 \\
Me exaspero fácilmente con los pacientes & 32 \\
Estoy menos comprometido con & \\
mis pacientes (p. ej., escuchar & \\
activamente y responder) & 29 \\
Soy menos amigable con los pacientes & 24 \\
Estoy menos motivado para ser cuidadoso & \\
al tomar los datos del paciente (p. & \\
ej., su historia clínica y el llenado de & \\
su registro electrónico de salud) & 14 \\
Expreso mi frustración frente a los pacientes & 14 \\
Cometo errores que antes no cometía & 5 \\
Cometo errores que podrían & \\
lastimar a los pacientes & \\
\hline
\end{tabular}

veces, aunque te empieces a dar cuenta no lo quieres aceptar o piensas que no te queda otra que seguir así (para los que nunca lo han sentido, la inmensa mayoría no pide ayuda).

Estar exhausto emocionalmente se conjuga con la percepción de ser menos eficientes en el trabajo; es difícil de darse cuenta porque en general es gradual. Lo que sí es de tomar en cuenta es que cada vez es más frecuente. En parte se debe a las demandas cada vez más exigentes de los sistemas de salud, pero donde hay poca autonomía y control. Este desgaste tiene en realidad consecuencias significativas para el profesionista, para sus pacientes, para su grupo de trabajo, para su familia, etc. Desde luego que produce anhedonia, ansiedad, trastornos del sueño, abuso de substancias, depresión, problemas de cognición (desmemorias, lentitud de pensamiento, desenfoques, errores más frecuentes) y de conducta (irritabilidad), resentimientos, procrastinación, sensación de aburrimiento y desilusión, mareos, ideación suicida, errores médicos, palpitaciones, falta de aire, dolores de pecho, de cabeza, fatiga crónica, debilidad del aparato inmune, dolores de estómago, falta de apetito, sensación de "estar hasta el gorro", pocas ganas de ir a trabajar, "Sunday night blues", etc.

Se conoce que 15\% de los médicos desarrolla un problema de abuso de substancias y/o problemas psiquiátricos. Todo esto empieza desde que somos estudiantes de medicina. Por ello en Estados Unidos ya hay muchos programas que se han realizado para tratar de proteger a los estudiantes. De hecho, la ocurrencia de suicidios entre médicos ha sido uno de los grandes promotores de que se estudie el desgaste profesional y se hayan propuesto tratamientos para evitarlo. Varias asociaciones médicas en Estados Unidos se han unido para armar programas que alivien el estrés y el desgaste de los médicos.

La Dra. Claire Zilber, ${ }^{6}$ experta en situaciones de ética, ha alertado de las consecuencias éticas y legales de los errores médicos cometidos debido a las ineficiencias producidas por esta condición. Desafortunadamente los

Tabla 5: ¿Su depresión afecta sus relaciones con colegas o el personal?

\begin{tabular}{lc}
\hline & $\%$ \\
\hline $\begin{array}{l}\text { Estoy menos comprometido con } \\
\text { mis colegas/trabajadores (p. ej., } \\
\text { escuchar activamente y responder) }\end{array}$ & 42 \\
Me exaspero más fácilmente con & \\
mis colegas/trabajadores & 42 \\
Expreso mi frustración frente a & \\
mis colegas/trabajadores & 37 \\
Soy menos amigable con mis & \\
colegas/trabajadores & 36 \\
Mi depresión no afecta mi & \\
comportamiento en el trabajo & 22 \\
Llego tarde al trabajo & \\
Cometo errores que antes no cometía & 21 \\
Me tomo más tiempo para comer & 11 \\
Cometo errores que podrían lastimar & 9 \\
a mis colegas/trabajadores & 2 \\
\hline
\end{tabular}

Tabla 6: ¿Qué contribuye al desgaste de los médicos?

\begin{tabular}{ll}
\hline & $\%$ \\
\hline $\begin{array}{l}\text { Demasiadas tareas burocráticas (p. } \\
\text { ej., hacer estadísticas, papeleo) }\end{array}$ & 56 \\
$\begin{array}{l}\text { Pasar demasiadas horas en el trabajo } \\
\text { Falta de respeto de los jefes/ }\end{array}$ & 39 \\
administradores, colegas o trabajadores & 26 \\
Creciente práctica computarizada & \\
(registros electrónicos de salud) & 24 \\
Bajo salario & \\
Falta de control/autonomía & 24 \\
Sentirse como un simple engrane & 21 \\
dentro de una máquina & 20 \\
Falta de respeto de los pacientes & \\
Regulaciones gubernamentales & 16 \\
Ingresos decrecientes & 16 \\
Énfasis en las ganancias & 15 \\
sobre los pacientes & 15 \\
Requisitos para mantener la certificación & 12 \\
\hline
\end{tabular}


Tabla 7: ¿Qué reduciría el desgaste?

\begin{tabular}{lc}
\hline & $\%$ \\
\hline Aumentar el sueldo para evitar & 35 \\
el estrés financiero & 31 \\
Horarios y llamados de & 27 \\
trabajo más cómodos & 24 \\
Reducir las regulaciones del gobierno & 23 \\
Cargas de pacientes más razonables & 23 \\
Mayor control/autonomía & \\
Mayor respeto de los jefes/ & 23 \\
administradores, colegas o trabajadores & 20 \\
Más vacaciones pagadas & 19 \\
Horarios más flexibles & 19 \\
Más personal de apoyo & \\
Énfasis en los pacientes & 12 \\
sobre las ganancias & 8 \\
Mayor respeto de los pacientes & 8 \\
Actitudes más positivas de los colegas & \\
Más oportunidades de desarrollo & 5 \\
académico/profesional & \\
Pareja o esposo(a) empático & \\
y que brinda apoyo & \\
\hline
\end{tabular}

pocos estudios realizados hablan desfavorablemente de los médicos que tienden a ser deshonestos y a engañar. Su pequeño artículo es muy elocuente en las consecuencias desastrosas que pueden ocurrir.

Es de vital importancia — repito de vital importanciacuidarnos bien para estar bien, con nosotros mismos, con nuestras familias, amigos, colegas y con nuestros pacientes.

Este problema generalmente está "bajo del agua", es decir, no es muy visible. Hay que quitar como dije hace un minuto, pero aun la Asociación Médica Americana en marzo del 2017 la clasificó como una crisis del sistema de salud.

¿Qué ayuda a bajar el desgaste? (Tabla 7).

¿Qué hacemos naturalmente? (Tabla 8).

\section{TRATAMIENTOS}

Se ha visto que los mejores tratamientos son sistémicoscorporativos, o sea, que si todos alrededor no están conscientes y trabajan terapéuticamente, la solución del problema llevará más tiempo. Generalmente las instituciones no ofrecen apoyos y menos de manera privada.

Desgraciadamente las soluciones no están muy bien establecidas, sin embargo, sí se conocen ciertas técnicas útiles. Algunas intervenciones son de sentido común, pero como sabemos el sentido común, es lo último que se utiliza.

Lo primero es descansar; esto se dice muy fácil, pero es el problema primordial. Para conseguir esto se requiere de un cambio de carácter, lo cual es muy difícil de conseguir: ser menos heroico y estoico, dimensionar las capacidades y medir los retos, encontrar ayuda dentro del equipo de trabajo y sentirse cómodo en delegar responsabilidades, esperando que haya alguien que las tome.

Para reducir el estrés tenemos que quitar una o dos actividades del día, tomar descansos, tomarse unos cuantos minutos muchas veces ayuda bastante. Todos los días debemos de tener periodos de relajación y de risa. Yo me di cuenta que ya estaba en el hoyo cuando borraba de inmediato los dos chistes muy cortos que desde hace muchos años programé para que me llegaran a mi computadora todos los días. No me daba yo el tiempo, en realidad eran unos 30 segundos para leerlos.

Las intervenciones usadas varían entre ser sistémicas o individuales, preventivas o de reducción de síntomas, presenciales o en línea, cortas o largas, etc. Hay que, desde luego, reforzar la resiliencia: el uso de métodos cognitivo/ conductuales, el poder aumentar la comunicación emotiva, la reducción de niveles de estrés y, desde luego, el uso de respiración saludable y sobre todo de técnicas de meditación y mindfulness. Nada más, como un pequeño abordaje cognitivo-conductual es dar la dimensión real a los problemas que uno enfrenta; se debe de regular el ciclo sueño-vigilia, los periodos recreativos emocionalmente satisfactorios, ejercicio adecuado, dietas saludables, terapias grupales o individuales. En ocasiones el uso de psicofármacos es necesario. El cambio de vida, rutina, horarios, demandas profesionales es esencial. El sistema, instituciones y los compañeros deben de cooperar. Las guardias médicas no deben de ser muy pesadas.

Existen muy pocos estudios de las intervenciones contra el burnout: se reportó un estudio en JAMA Medicina Interna del 1 de febrero del 2017 y otro estudio en Lancet el 5 de noviembre de 2016.

Las mujeres son las más afectadas: 55\% frente a $46 \%$ en hombres. Desde luego mucho de ello es porque están embarazadas o tienen hijos pequeños y siguen trabajan-

Tabla 8: ¿Cómo enfrentan los médicos el agotamiento?

\begin{tabular}{lc}
\hline & $\%$ \\
\hline Ejercicio & 50 \\
Hablar con parientes o amigos cercanos & 46 \\
Dormir & 42 \\
Aislarse de los otros & 36 \\
Tocar o escuchar música & 36 \\
Comer comida chatarra & 33 \\
Beber alcohol & 22 \\
Darse un atracón & 20 \\
Otros & 10 \\
Fumar o usar productos con nicotina & 3 \\
Toma tranquilizantes farmacéuticos & 2 \\
Fuma o consume productos con marihuana & 1 \\
\hline
\end{tabular}


do. En estas situaciones las mujeres tienen tres veces más oportunidades de quitarse la vida que los hombres, según la Dra. Kristin Budde (Figura 7).

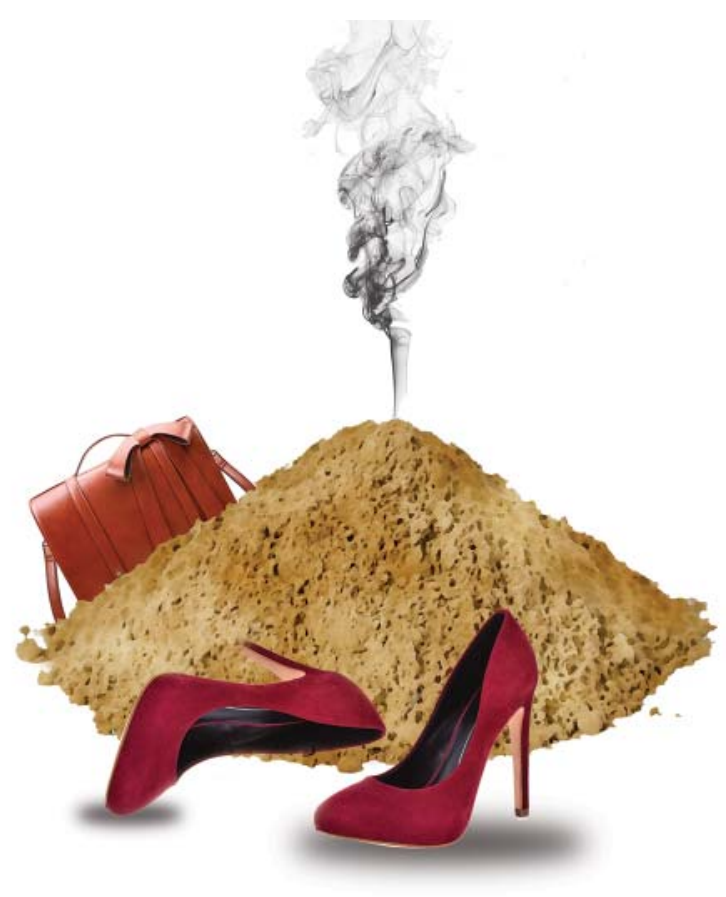

Figura 7.
En algún momento vas a tener que escoger en esta encrucijada entre balacear tu vida o echarla a perder.

Tomar café como antioxidante, desde luego tienes que comer bien y tratar de socializar con amistades que les gusta divertirse, que sean positivos y con los que te sientes cómodo.

Sólo para repetir una vez más hay que identificar que existe distrés y de donde proviene. Comunicarlo; ser espontáneo, romper rutinas (algo casi imposible de hacer), probar novedades, mantenerte sano, ejercicio, etc.

- $\quad$ Repaso de síntomas y desencadenantes (Figura 8)

- Muerte (Figura 9)

- $\quad$ Perspectiva neuroendocrinológica (Figura 10)

¿Quién pide ayuda? (Tabla 9) Más allá del yoga, existe otra filosofía milenaria para encontrar la felicidad y el equilibrio, y de la que muchas celebrities dentro y fuera de nuestro país ya se han declarado fieles seguidoras. Se trata del Ho'oponopono, un método hawaiano de meditación que, básicamente, nos enseña a "hacer las paces" con nosotros mismos y con los demás.

Para ello, se sirve de un sencillo mantra que podemos aplicar para resolver cualquier problema de nuestra vida cotidiana, y que consiste en repetir cuatro palabras mágicas: "Lo siento, perdóname, te amo y gracias". Algo que, reconozcámoslo, no todos estamos acostumbrados a poner en práctica con mucha frecuencia.

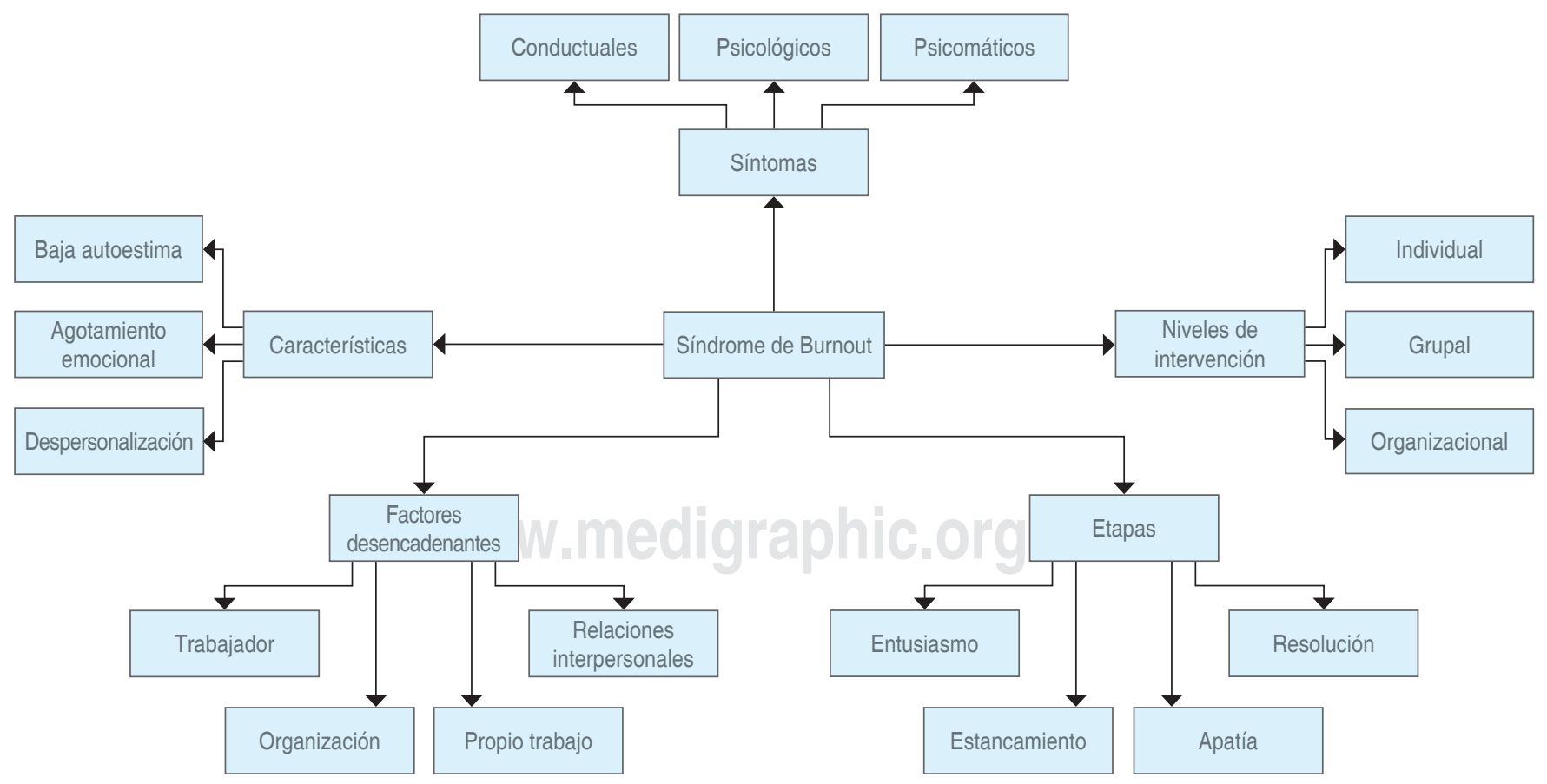

Figura 8. 


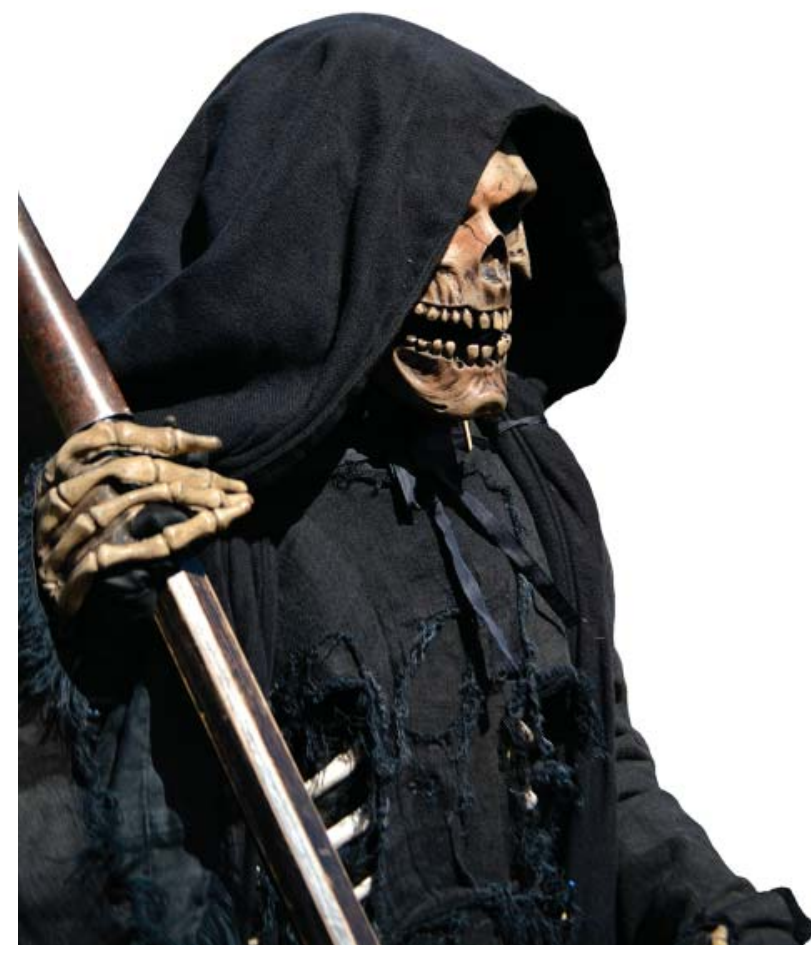

Figura 9.

Mindfulness es cada vez más estudiada por los neurocientíficos y cada vez es más reconocida como un tratamiento que, aunque simple es muy útil. Es la regulación mental de la atención en el aquí y en el ahora. De esta manera tenemos una conciencia más clara y controlada de nuestros procesos mentales; está caracterizada por la curiosidad, la apertura y la aceptación de las experiencias que son vividas en ese momento. Se debe de quitar valores de juicio dando paso a la aceptación. Se debe de tener una postura cómoda, un foco de atención y actitud de despeje de lo cotidiano.

Mindfulness reduce la activación autonómica, aumenta la regulación emocional y mejora el sueño; la resiliencia aumenta, así como los sentimientos y afectos positivos para tener un mejor balance, desarrollo personal y enganche. Es la práctica de estar atento, aceptando las experiencias en el momento presente. Cada vez esta práctica está siendo reconocida para disminuir el estrés, aumentar el autoconocimiento y la autoconciencia, además del bienestar personal que aumenta y promueve las relaciones significativas. Se basa en una capacidad natural y habilidades que se van fortaleciendo con la práctica mental.

Programas de Mindfulness se han diseminado en todo el mundo como pólvora. Para sólo ejemplificar uno que se implementa en el hospital en que yo me entrené como Psiquiatra, Psicogeriatría y Paidopsiquiatra en Baltimore, entonces Ilamado el Hospital Sheppard and Enoch Pratt y que tiene una unidad "pipirisnais", que se llama el "retreat", manejado por Trina Lion, el cual ofrece este tratamiento tanto a pacientes como el personal que ahí trabaja, con muy buenos resultados. ${ }^{7}$ En el caso de los médicos, es una herramienta muy poderosa que aumenta la resiliencia frente al estrés y disminuye el desgaste profesional. Aunque muchas veces lo hacemos sin darnos cuenta, el problema es que lo hacemos muy poco tiempo y este tiempo es aplastado por la prisa que tenemos en hacer cosas.

Estamos acostumbrados a tratar de hacer muchas cosas al mismo tiempo sin darnos cuenta del aquí y del ahora. El ser entrenado como médicos muchas veces nos demanda que nos ignoremos a nosotros mismos. Así, nunca nos damos cuenta de nuestro bienestar. Lo que hacemos los médicos rutinariamente sería considerado en otras industrias como abusivo y hasta como ilegal. Estamos acostumbrados a ser omnipotentes, heroicos y estoicos.

Una definición de "bienestar" es el alcanzar un estado satisfactorio de existencia donde hay salud, felicidad y prosperidad. Ya se ha comprobado que la práctica de mindfulness trae muchos beneficios tanto físicos como mentales y desde luego se reduce el síndrome de desgaste profesional.

Se ha comprobado que usar estas técnicas induce en la materia blanca cambios en el cíngulo anterior. También aumenta la densidad de la materia gris.

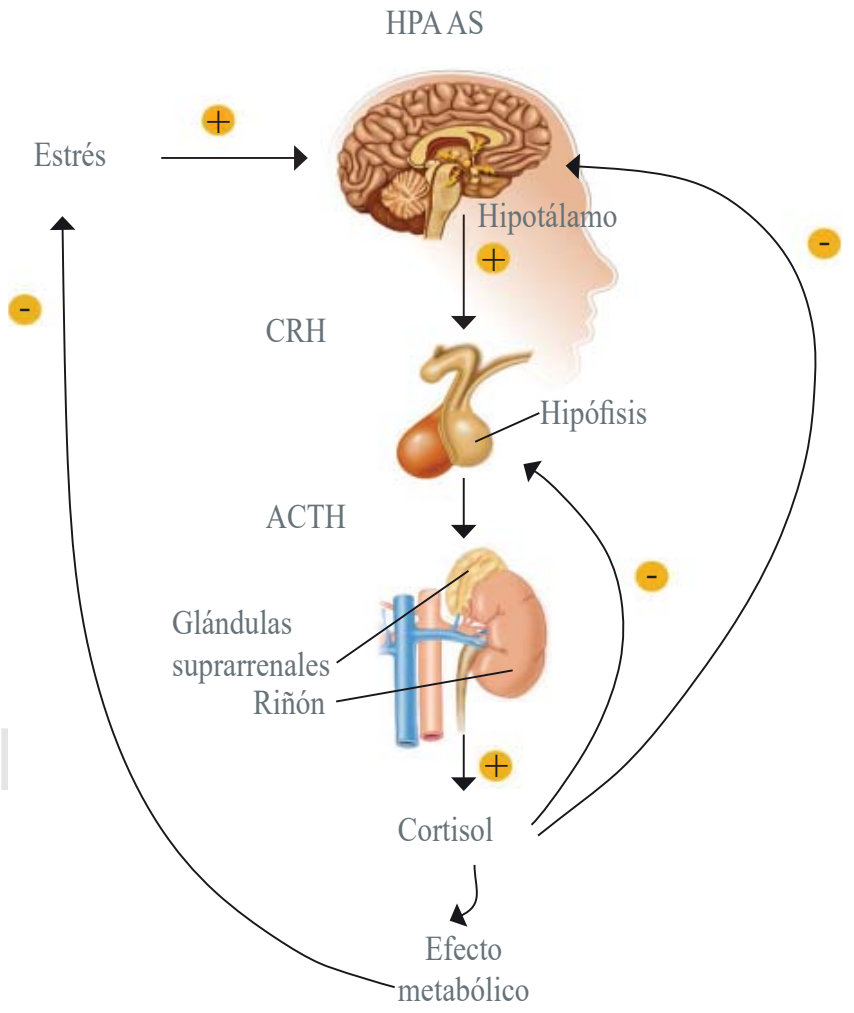

Figura 10. 
Tabla 9: ¿Qué médicos son más propensos a buscar ayuda?

\begin{tabular}{ll}
\hline Especialidad médica & $\%$ \\
\hline Psiquiatría & 40 \\
Cirugía Plástica & 35 \\
Salud Pública y Medicina Preventiva & 33 \\
Pediatría & 33 \\
Obstetricia/Ginecología & 32 \\
Medicina Familiar & 31 \\
Dermatología & 30 \\
Medicina Crítica & 29 \\
Patología & 29 \\
Medicina Física y Rehabilitación & 29 \\
Enfermedades Infecciosas & 28 \\
Neurología & 27 \\
Medicina Interna & 24 \\
Medicina de Emergencia & 24 \\
Alergias e Inmunología & 23 \\
Oftalmología & 23 \\
Anestesiología & 23 \\
Radiología & 22 \\
Reumatología & 22 \\
Gastroenterología & 22 \\
Oncología & 22 \\
Otorrinolaringología & 22 \\
Cirugía General & 21 \\
Diabetes y Endocrinología & 21 \\
Medicina Pulmonar & 20 \\
Ortopedia & 20 \\
Nefrología & 20 \\
Urología & 18 \\
Cardiología & 17 \\
\hline &
\end{tabular}

Desde un punto más profundo algunas veces se deben de usar técnicas psicoanalíticas para disminuir las resistencias como la negación o los problemas de carácter como el perfeccionismo.

Parte de lo que se recomienda — como ya lo mencioné hace unos momentos - es aumentar la resiliencia. La resiliencia es un proceso de adaptación dinámico y flexible frente a los cambios en la vida y que puede servir como factor protector en contra de la ansiedad y el distrés emocional, además de prevenir enfermedades mentales.

Se recomienda que los médicos compartan sus experiencias con otros colegas, ya que esto reduce el aislamiento profesional.

Yo ya tengo varios años de respirar profundo pausadamente en una forma automática cuando empiezo a sentir estrés. Generalmente desde la primera sesión que tengo con mis pacientes les enseño brevemente cómo respirar para que les disminuya un poco el estrés. Les dejo ver cómo yo respiro profundo y les confieso que esto es un método que uso para disminuir el estrés que me producen las cosas que ellos me cuentan.
Aunque parezca repetitivo, tenemos que darnos cuenta de que el balance entre la vida común y corriente y el trabajo requiere de manejo del tiempo, eliminando las cosas que no son esenciales para tener prioridades o una lista de las cosas que son posibles de hacer. Realizar un asesoramiento y realineación de metas, habilidades, y pasiones en el trabajo. Se necesita tener un sueño adecuado, comer sanamente y hacer ejercicio regularmente. Se debe de incluir tiempos recreativos y de relajación como puede ser un deporte o yoga, meditación o un hobby. Se debe de construir un sistema de apoyo personal y de trabajo. Tener acceso actividades culturales y/o espirituales. Tómese unas vacaciones.

Debemos de entender que primero nos debemos de dar a nosotros mismos para poder dar a los demás. Tenemos que tener una red de apoyo y convivencia.

Para aguantar la vida tan estresante que tenemos, requerimos de tener salud y energía, positivismo, habilidades para resolver problemas, habilidades sociales y apoyos externos; humildad para pedir ayuda.

Desde el punto de vista organizacional tienes que cambiar el desgaste (estado exhausto, el cinismo, y la ineficiencia) hacia el enganche (energía, vigor, dedicación).

Tienes que darte cuenta del problema y evaluarlo claramente. Acercarte a las autoridades para describir el problema. Cultivar el sentido de comunidad en el trabajo. Usar incentivos inteligentemente. Alinear los valores. Promover flexibilidad tanto en el trabajo como en la vida privada.

\section{REFERENCIAS}

1. DSM-5.

2. ICD-10.

3. Young KD, Vega CP. What is the cost of physician burnout in the united states? Medscape. 2019. Available in: https://www.medscape.org/viewarticle/915596?nlid=131048_2804\&src $=$ wnl_ cmemp_190816_mscpedu_psyc\&uac $=61465 \bar{F} Z \& i m p I D=2062 \overline{7}$ $19 \&$ faf $=1$

4. Block NT. Burnout cultural shift needed. Psychiatric News. 2017; 52 (23).

5. Guille C. Overcoming Burnout. Psychiatric News. 2017; 52 (17).

6. Wick P. Tools for countering burnout mirror those for aging well as a physician. Psychiatric News. 2018; 53 (2).

7. Medscape National Physician Burnout \& Depression Report. 2018. Available in: https://www.medscape.com/slideshow/2018lifestyle-burnout-depression-6009235?src=ppc_google_rem_burnout_ous

8. Sorgen C. Mindful meditation useful to avoid burnout, promote wellness. Psychiatric News. 2018; 53 (1).

9. Zilber C. Burnout and wellness from an ethics perspective. Psychiatric News. 2018; 53 (1).

10. Budde K. Babies and burnout: should being a mom mean giving up ambition? Psychiatric News. 2018; 53 (5).

\section{LECTURAS RECOMENDADAS}

Intervenciones Organizacionales

- AMAs "Step Forward" Practice Improvement Strategies: https:// www.stepforward.org/ 


\section{Intervenciones para médicos}

— 10 TED Talks on Burnout: https://www.ted.com/playlists/245/talks for_when_you_feel_totall

- The Happy Secret to Better Work: https://ted.com/talks/shawn ancor the happy_secret to better_work

- Health and Wellness: http://www.ismanet.org/doctoryourspirt/ HealthWellnessSolutions.html

- Free Mindfulness Apps Worthy Your Attention: https://www.mindful.org/free-mindfulness-apps-worthy-of-your-attention/

\section{De la Asociación Psiquiátrica Americana}

- https://www.psychiatry.org/psychiatrists/practice/well-being-andburnout/well-being-resources

- www.psychiatry.org/burnou

- Escala para medir el desgaste. Oldenburg Burnout Inventory.

- https://www.psychiatry.org/psychiatrists/practice/well-being-andburnout/assess-yourself

- Métodos estandarizados para hacer filtros. 